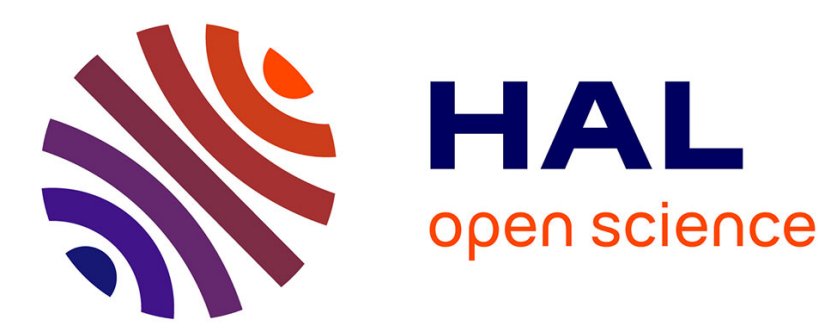

\title{
Phase diagram of boron-doped diamond revisited by thickness-dependent transport studies
}

J. Bousquet, T. Klein, M. Solana, L. Saminadayar, C. Marcenat, E. Bustarret

\section{To cite this version:}

J. Bousquet, T. Klein, M. Solana, L. Saminadayar, C. Marcenat, et al.. Phase diagram of borondoped diamond revisited by thickness-dependent transport studies. Physical Review B, 2017, 95 (16), pp.161301. 10.1103/PhysRevB.95.161301 . hal-01348500

\section{HAL Id: hal-01348500 \\ https://hal.science/hal-01348500}

Submitted on 24 Jul 2016

HAL is a multi-disciplinary open access archive for the deposit and dissemination of scientific research documents, whether they are published or not. The documents may come from teaching and research institutions in France or abroad, or from public or private research centers.
L'archive ouverte pluridisciplinaire HAL, est destinée au dépôt et à la diffusion de documents scientifiques de niveau recherche, publiés ou non, émanant des établissements d'enseignement et de recherche français ou étrangers, des laboratoires publics ou privés. 


\title{
Phase diagram of boron-doped diamond revisited by thickness-dependent magneto-transport studies
}

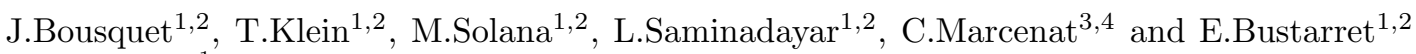 \\ 1 Université Grenoble Alpes, Institut NEEL, F-38042 Grenoble, France \\ 2 CNRS, Institut NEEL, F-38042 Grenoble, France \\ 3 Université Grenoble Alpes, INAC-SPSMS, F-38000, France and \\ 4 CEA, INAC-Pheliqs, F-38000, France
}

(Dated: July 24, 2016)

\begin{abstract}
We report on a detailed study of the electronic properties of a series of boron-doped diamond epilayers with dopant concentrations ranging from $1.10^{20}$ to $3.10^{21} \mathrm{~cm}^{-3}$ and thicknesses $\left(d_{\perp}\right)$ ranging from $2 \mu \mathrm{m}$ to $8 \mathrm{~nm}$. By using well-defined mesa patterns that minimize the parasitic currents induced by doping inhomogeneities, we have been able to unveil a new phase diagram differing from all previous reports. We show that the onset of superconductivity does actually not coincide with the metal-insulator transition in this system. Moreover a dimensional crossover from $3 \mathrm{D}$ to $2 \mathrm{D}$ transport properties could be induced by reducing $d_{\perp}$ in both the metallic non-superconducting and superconducting epilayers, without any reduction of $T_{c}$ with $d_{\perp}$ in the latter.
\end{abstract}

PACS numbers: 74.60.Ec, 74.60.Ge

The discovery of superconductivity in boron-doped diamond was first reported in 2004 by Ekimov et al. [1] in high pressure high temperature polycrystalline samples and was rapidly confirmed in both polycrystalline [2] and (100)-oriented single crystal [3] films grown by Microwave Plasma enhanced Chemical Vapor Deposition (MPCVD). Various experimental works including (i) the softening and broadening of the Brillouin zone center phonon mode at the transition [4], (ii) the existence of an isotopic effect on $T_{c}$ [5] or (iii) the observation of a fully open superconducting gap $(\Delta)$ with $2 \Delta(0) / k_{B} T_{c}$ being close to the standard BCS weak coupling value [6] then provided strong experimental insights in favor of a standard electron-phonon coupling mechanism. With critical temperatures $\left(T_{c}\right)$ rising up to $\sim 10 K[7,8]$ despite very small carrier concentrations $\left(\sim 10^{21} \mathrm{~cm}^{-3}\right)$, this discovery revived the interest for "superconducting semiconductors" as promising candidates for possible new conventional - high temperature superconductors (for a review see $[9,10])$.

However several questions remain open. Indeed, early studies $[7,11]$ suggested that $T_{c}$ remained surprisingly large down to the metal-insulator transition (MIT) and that this system could hence provide new insights on the still highly debated - mechanism leading to the insulatorsuperconductor transition (for a review, see [12]). A sharp peak in the resistivity prior to the superconducting transition has for instance been reported in granular diamond films and interpreted as a signature of a metal Bosonic insulator - superconductor transition [13]. Moreover, it has been suggested that the critical temperature scaled as $T_{c} \propto\left(n_{B} / n_{c}^{M I T}-1\right)^{1 / 2}[11]$ in striking contrast with the exponential dependence expected from the standard McMillan expression $\left(n_{B}\right.$ being the Boron content and $n_{c}^{M I T}$ the critical concentration corresponding to the MIT) and that $T_{c}$ strongly - and anomalously - depended on the thickness of the epilayers [14]. All those measurements, clearly indicating a strong interplay between the onset of superconductivity and the MIT, pointed out this system as an interesting platform to study the interaction between electronic correlations, disorder and superconductivity in the vicinity of the insulator-superconductor transition.

We present here a detailed study of the electronic properties of boron-doped diamond epilayers in the vicinity of the MIT. Both the influence of the doping content and of the epilayer thickness have been revisited. The main novelty of our work is the use of well defined mesa patterns (see Fig.1) that enabled us to control the current distribution within the sample, and hence to minimize the effects associated to parasitic current paths induced by doping inhomogeneities. We have then been able to unveil the existence of a new metallic non-superconducting state separating the insulating (for $n_{B} \leq n_{c}^{M I T} \sim 3 \pm 1$. $10^{20} \mathrm{~cm}^{-3}$ ) from the superconducting states (for $n_{B} \geq$ $\left.n_{c}^{S} \sim 11 \pm 2.10^{20} \mathrm{~cm}^{-3}\right)$. We show that disorder, quantified by the Ioffe-Regel parameter [15] $k_{F} l$ (where $k_{F}$ is the Fermi wave vector and $l$ the mean free path) might play a role as the onset of superconductivity is observed for $k_{F} l \sim 1$, but, in contrast to [14], we did not observe any reduction of $T_{c}$ with the sample thickness $\left(d_{\perp}\right)$ down to $8 \mathrm{~nm}$. Moreover, we show that superconductivity is very robust to dimensionality as it can be observed in both $3 \mathrm{D}\left(L_{T}<d_{\perp}\right)$ and $2 \mathrm{D}\left(L_{T}>d_{\perp}\right)$ regimes $\left(L_{T}\right.$ being the thermal diffusion length).

A series of Boron-doped epilayers was grown by MPCVD on top of hundred nanometer-thick NonIntentionally Doped (NID) layers deposited on IIa- or Ib-type [100]-oriented diamond substrates [17]. The total pressure in the vertical silica tube reaction chamber was set to 33 or 50 torr in order to stabilize the sample temperature to $\sim 910^{\circ} \mathrm{C}$ and $\sim 830^{\circ} \mathrm{C}$ during the growth of NID and doped-layer respectively. After a first exposure of the substrate to a pure hydrogen plasma, methane $\left(\mathrm{CH}_{4} / \mathrm{H}_{2}=1 \%\right.$ molar ratio $)$ has been added for the buffer layer deposition. The gas 
mixture has been complemented by addition of oxygen $\mathrm{CH}_{4} / \mathrm{O}_{2} / \mathrm{H}_{2}(0.75 \%, 0.25 \%)$ to reduce the residual boron incorporation and the surface roughness [18]. Finally, gas mixtures with various concentrations of diborane were used for the B-doped epilayers. The substrate was placed either within or at the vicinity of the plasma ball and the growth parameters corresponding to these two positions are summarized in table 1 . The thickness of the epilayers was controlled by varying the growth time and checked by ellipsometric measurements [16]. A total number of 26 samples, with doping level ranging between $1.10^{20}$ and $3.10^{21} \mathrm{~cm}^{-3}$ were grown with thicknesses ranging from 8 $\mathrm{nm}$ to $2 \mu \mathrm{m}$.

Transport measurements have been performed between $300 \mathrm{~K}$ and $3 \mathrm{~K}$ using a Quantum Design Physical Properties Measurements System and down to $50 \mathrm{mK}$ by adding a homemade adiabatic demagnetization refrigeration stage on the former setup and/or by using a standard dilution fridge. Four contact measurements have been carried out on each epilayer first using only silver pasted top contacts (similar to previous studies [11]) and subsequently using well-controlled mesa patterned Hallbars (see sketch in the inset of Fig.1) delineated from the surrounding doped layers by using $\mathrm{O}_{2}$ plasma treatment (with $\mathrm{Ti} / \mathrm{Pt} / \mathrm{Au}$ metallic pads). The temperature dependence of the renormalized conductivity of selected characteristic layers, measured on the mesa patterns, are reported Fig.1.

It is important to note that the Boron concentrations discussed throughout this work have been deduced from Hall effect measurements. Even though anomalously small Hall coefficients have been previously reported $[7,11]$ (hindering any reliable determination of the doping concentration from these measurements), the hole concentrations deduced from our measurements performed on mesa patterned geometries are fully consistent with those deduced from ellipsometry measurements [16] and with the boron content determined by Secondary Ion Mass Spectroscopy measurements (SIMS, see [11] for details) performed on selected samples. Note that we have also obtained significantly reduced Hall coefficients when measuring the Hall effect with contacts directly deposited on the top of the samples without further mesa patterning, hence clearly stressing out the necessity of a systematic delineation of the current paths in order to obtain relevant results.

Similarly, the non superconducting metallic phase could not be observed when measuring the resistivity of the same films with pasted silver pads without further mesa-patterning (as done in [11]). We then obtained a systematic decrease of the critical temperatures in all samples upon mesa-patterning. Not any superconducting transition could be observed for $n_{c}^{M I T} \leq n_{B} \leq n_{c}^{S}$, unveiling the existence of a non superconducting metallic state in boron doped diamond. On several films, twelve Hall bars have been patterned all over the surface in order to map the distribution of the electronic properties. For $n_{B} \gtrsim n_{c}^{S}$, this mapping procedure revealed the presence
TABLE I: Total gas flow rate, $\mathrm{CH}_{4} / \mathrm{H}_{2}$ molar ratio, growth rate (in $\mathrm{nm} / \mathrm{min}$ ) and boron to carbon concentration ratio used during the growth process of the epilayers. The substrate was placed either within the plasma ball (position 1) or at its vicinity (position 2).

\begin{tabular}{ccccc}
\hline \hline position & $\begin{array}{c}\text { flow rate } \\
\text { sccm }\end{array}$ & $\begin{array}{c}\mathrm{CH}_{4} / \mathrm{H}_{2} \\
\%\end{array}$ & $\begin{array}{c}\text { Growth rate } \\
\text { nm/min }\end{array}$ & $\begin{array}{c}\mathrm{B} / \mathrm{C} \\
\text { ppm }\end{array}$ \\
\hline 1 & 100 to 400 & 4 & $\sim 32$ & 400 to 2500 \\
2 & 2000 & 0.5 & $\sim 5$ & 6000 to 12000 \\
\hline \hline
\end{tabular}

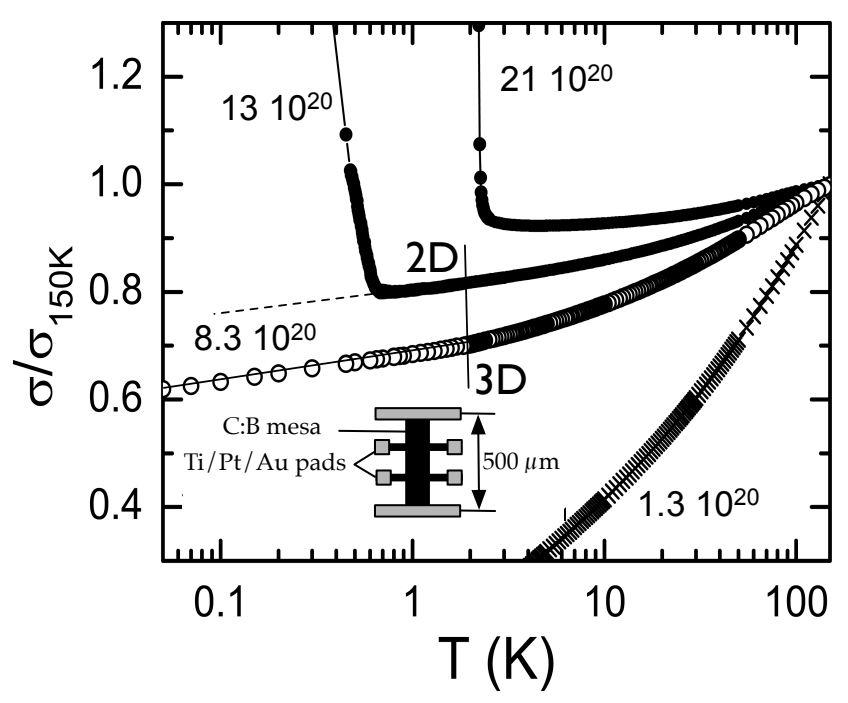

FIG. 1: Temperature dependence of the conductivity (renormalized to its value at $150 \mathrm{~K}$ ) in four boron doped diamond epilayers for the indicated carrier concentration (in $\mathrm{cm}^{-3}$ ). As shown, both superconducting (solid circles), metallic non superconducting (open circles) and insulating (crosses) samples can be obtained depending on the boron concentration (see text for details). Inset : sketch of the mesa structured Hall-bars patterned on the $3 \times 3 \mathrm{~mm}^{2}$ epilayers. A dimensional crossover separating $3 \mathrm{D}$ and $2 \mathrm{D}$ interaction regimes (vertical line) has been observed in the thinnest samples (see Fig.3 and text for details).

of incomplete transitions in some of the Hall bars located close to the sample edges, suggesting the presence of percolation paths with larger boron concentrations within the peripheral area.

After mesa-patterning, a superconducting transition has been observed on about half of the samples (see solid circles in Fig.1) whereas no drop of resistivity could be detected on the second half of the metallic samples down to $50 \mathrm{mK}$ (see open circles in Fig.1). Finally for the lowest Boron concentrations $\left(n_{B}<n_{c}^{M I T} \sim 3 \pm 1.10^{20}\right.$ $\mathrm{cm}^{-3}$ ), an insulating behavior with diverging resistivity at low $\mathrm{T}$ is recovered (see crosses in Fig.1). The critical temperatures (defined as the temperature at which the resistivity decreases to $90 \%$ of the normal state value) 


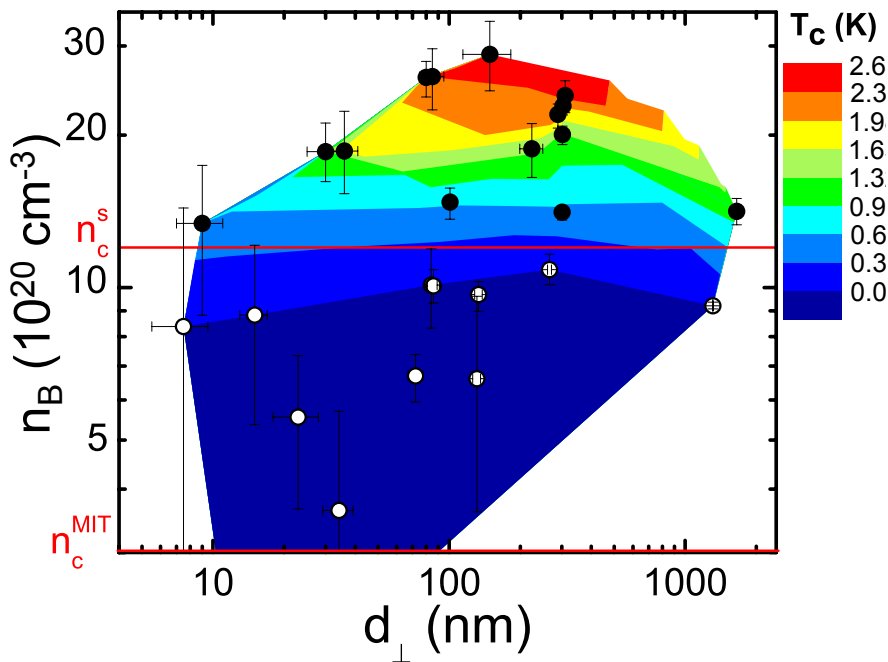

FIG. 2: (color on line) Critical temperature as a function of thickness $\left(d_{\perp}\right)$ and doping content $\left(n_{B}\right.$, deduced from Hall effect measurements) in C:B epilayers. A non superconducting metallic phase (open symbols) has been observed for $n_{c}^{M I T}<n_{B}<n_{c}^{S}$ (with $n_{c}^{M I T}=3 \pm 1.10^{20} \mathrm{~cm}^{-3}$ and $\left.n_{c}^{S}=1.1 \pm 0.2 .10^{21} \mathrm{~cm}^{-3}\right)$. This color plot clearly indicates that $T_{c}$ does not depend on the layer thickness (down to 8 $\mathrm{nm}$ ). Note that 2D like quantum interference effects have been observed in the thinest samples (see for instance Fig.3c and Fig.3d) showing that superconductivity can be obtained in both $3 \mathrm{D}$ and 2D like samples. Insulating layers obtained for for $n_{B} \leq n_{c}^{M I T}$ have not been reported.

of all layers are displayed in Fig.2 as a function of their thickness and doping concentration. As shown, $T_{c} \rightarrow 0$ for doping concentrations $n_{B} \leq n_{c}^{S}=11 \pm 2.10^{20} \mathrm{~cm}^{-3}$ revealing the presence of a metallic but non superconducting phase for $n_{c}^{M I T} \leq n_{B} \leq n_{c}^{S}$. The main result of this work is hence the observation of this metallic non superconducting phase, in striking contrast with previous reports $[7,11]$ which suggested on the contrary that the onset of superconductivity coincided with the MIT.

Although slightly lower, the $n_{c}^{M I T}$ value $\left(\sim 3 \pm 1.10^{20}\right.$ $\mathrm{cm}^{-3}$ ) obtained in this work is in reasonable agreement with previous reports (see [19] and references therein). This $n_{c}^{M I T}$ value well agrees with the Mott criterion $k_{F} a_{B} \sim 1$ which assumes that the transition is reached when boron-related hydrogenic states with Bohr radius $a_{B}(\sim 4 \AA[20])$ overlap (here introducing the Fermi wave vector $\left.k_{F}^{3}=3 \pi^{2} n_{B}\right)$. However, if correlation effects do indeed play a fundamental role, disorder-induced localisation effects also have to be taken into account. Indeed, the Ioffe-Regel parameter $k_{F} l \sim R_{Q} /\left[n_{B}^{0.33} \rho_{R T}\right]$ can be determined from the room temperature resistivity $\rho_{R T} \sim m^{*} / e^{2} n_{B} \tau$ [21] (introducing $R_{Q}=h / e^{2} \sim 25 \mathrm{k} \Omega$ ) and, as shown in Fig.4 (top panel), the as-deduced $k_{F} l$ values are on the order of 1 for $n_{B} \sim n_{c}^{S}$ clearly indicating that both correlation effects and disorder play a significant role in the MIT and possibly in the onset of

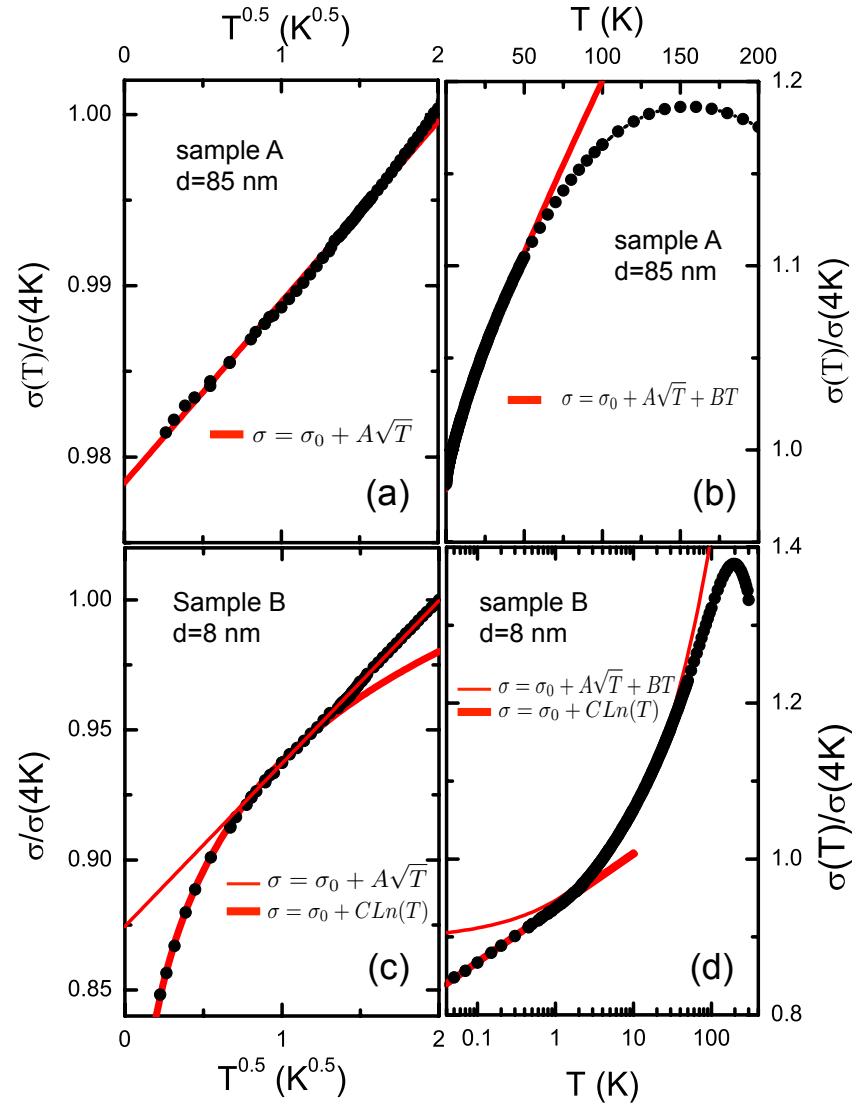

FIG. 3: (color online) Temperature dependence of the conductivity for two C:B samples with $n_{B} \sim 1.10^{21} \mathrm{~cm}^{-3}$ and $d_{\perp} \sim 85 \mathrm{~nm}$ (sample A) and $d_{\perp} \sim 8 \mathrm{~nm}$ (sample B). As shown, for $d_{\perp} \sim 85 \mathrm{~nm}, \sigma(T)$ can be well described by a $\sigma_{0}+A \sqrt{T}$ law at low temperature (thick solid (red) line in Fig.2a) as expected for 3D electron-electron interaction effects. At higher temperature, weak localisation effect have to be taken into account and $\sigma(T)=\sigma_{0}+A \sqrt{T}+B T$ (solid (red) line in Fig.2b, finally a standard metallic behavior is recovered above $\sim 150 \mathrm{~K}$ ). For $d_{\perp} \sim 8 \mathrm{~nm}, \sigma(T)$ is better described by a $\ln$ T term (thick (red) lines in Fig.2c and $2 \mathrm{~d}$ ) than by a $\sqrt{T}$ term (thin (red) lines), as expected in the $2 \mathrm{D}$ limit $\left(L_{T}>d_{\perp}\right)$.

\section{superconductivity.}

As expected, quantum corrections to the Drude resistivity then show up at low temperature and, as previously reported $[11,22]$, the temperature dependence of all metallic samples can be well described by a $\sigma(T)=$ $\sigma_{0}+\sigma_{E E I}(T)+\sigma_{W L}(T)[23]$ law for $T<50 \mathrm{~K}$ where the electron-electron interaction term $\sigma_{E E I}$ dominates below $\sim 5 K$ (see discussion below) and weak localisation corrections $\sigma_{W L} \sim B T^{p}$ drive the temperature dependence of the conductivity up to $\sim 50 \mathrm{~K}$. In this temperature range, inelastic scattering is expected to be dominated by electron-phonon interactions and $p=2$ or 1 depending on the influence of static impurities such as heavy impurities, defects or grain boundaries (see for instance the 


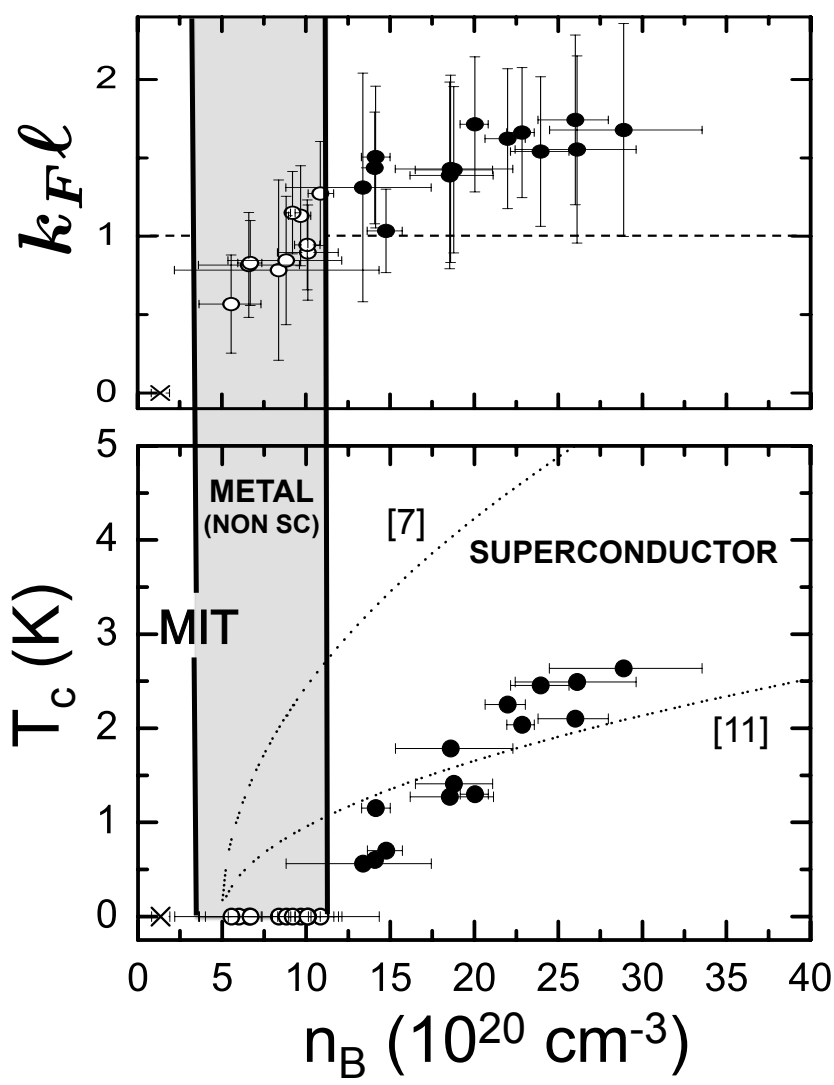

FIG. 4: Critical temperature (lower panel) and $k_{F} l$ value (upper panel) as a function of boron doping in C:B epilayers. The dotted line (lower panel) represents the doping dependence previously reported in [7] and [11].

discussion in [24]). Very reasonable fits to the data could be obtained taking $p \sim 1-1.5$ (see for instance solid (red) lines in Fig.3b and Fig.3d for $p=1$ in $d_{\perp} \sim 85 \mathrm{~nm}$ and $d_{\perp} \sim 8 \mathrm{~nm}$ epilayers respectively). We obtained $B \sim$ $0.5 \pm 0.1 \Omega^{-1} \mathrm{~cm}^{-1} \mathrm{~K}^{-1}$ (taking $p=1$ ) in all measured samples and the inelastic scattering time $\tau_{\Phi}$ is hence scaling as $1 / \tau_{\Phi} \sim\left[\pi R_{Q} B\right]^{2} . D T^{2} \sim\left[2.10^{9} \mathrm{~cm}^{-2} \mathrm{~K}^{-2}\right] . D T^{2}(\mathrm{D}$ being the diffusion constant $\sim 1-10 \mathrm{~cm}^{2} / \mathrm{s}$ ). Such a $D T^{2}$ scaling of the electron-phonon inelastic scattering rate has been previously reported in disordered systems [25] but a pertinent theoretical model for this dependence is still lacking (see discussion in [25]). Note that a significantly larger $\tau_{\Phi}$ value has been recently derived from (sub-) $\mathrm{THz}$ absorption measurements in the superconducting state of similar epilayers [24] but the origin of this discrepancy has still to be clarified.

Below $\sim 5 \mathrm{~K}$, electron-electron interactions clearly dominate and, as expected in 3D samples, the temperature dependence of the thick layers can be well described by a $\sigma(T)=\sigma_{0}+A \sqrt{T}$ law down to the lowest temperatures $(\sim 50 \mathrm{mK}$, see Fig.3a). However, in the thinnest samples, clear deviations from this law are observed (see Fig.3c for $d_{\perp} \sim 8 \mathrm{~nm}$ ) and the temperature dependence is then better described by a $\sigma_{E E I} \propto \ln T$ term for $T \lesssim 2$
$\mathrm{K}$ (see Fig.3c), as expected in 2D materials. Indeed, the thermal coherence length $L_{T}(T) \sim 1 /\left[\pi R_{Q} A \sqrt{T}\right] \sim$ $20[\mathrm{~nm}] / \sqrt{T}\left(A \sim 6 \pm 2 \Omega^{-1} \mathrm{~cm}^{-1} \mathrm{~K}^{-1 / 2}\right.$ in all measured samples), and a dimensional crossover is expected to be reached for $L_{T}\left(T_{c r}\right) \sim d_{\perp}$ i.e. for $T=T_{c r} \sim 5 \mathrm{~K}$ in the $d_{\perp}=8 \mathrm{~nm}$ sample, in very reasonable agreement with the observed behavior.

As shown in Fig.1 (see also Fig.2) superconductivity is observed in both 2D and 3D interaction regimes, clearly showing that superconductivity is robust to this dimensional crossover. Note that we did not observe any significant decrease of the critical temperature with $d_{\perp}$ (see Fig.2), in striking contrast with the clear decrease of $T_{c}$ reported by Kitagoh et al. [14]. We believe that such an apparent decrease can be induced by doping inhomogeneities. Indeed, interaction effects are expected to lead to a reduction of the critical temperature in thin samples [26]. However, for $R_{\square} /\left[\pi R_{Q}\right]<<1$, one expects $\Delta T_{c} / T_{c, 0} \approx-R_{\square} / R_{c}$ where $R_{\square}$ is the square resistance, $R_{c}=6 R_{Q} / \gamma^{3}, e^{\gamma}=\left[\hbar / \tau^{*}\right] /\left[k_{B} T_{c, 0}\right]$ and $\tau^{*}=\max \left\{\tau, \tau\left(d_{\perp} / l\right)^{2}\right\}[27]$ and, as $d_{\perp}>>\sim 4 \AA$, $\gamma \rightarrow 1\left(\tau \sim 10^{-15} \mathrm{~s}\right)$ so that no significant reduction of $T_{c}$ due to interaction effects is expected in our case.

In conclusion, we have revisited the phase diagram of boron doped diamond epilayers. A new diagram has been obtained by using well-defined mesa patterns that minimize the parasitic currents induced by doping inhomogeneities. This new phase diagram is displayed in Fig.4. The presence of a metallic - non superconducting - phase for $n_{c}^{M I T} \leq n_{B} \leq n_{c}^{S}$, with $n_{c}^{M I T}=3 \pm 1$. $10^{20}$ and $n_{c}^{S}=11 \pm 2.10^{20} \mathrm{~cm}^{-3}$ has been unveiled. As shown, the critical temperature rapidly rises for $n_{B} \geq n_{c}^{S}$ reaching $T_{c} \sim 2-3 \mathrm{~K}$ for $n_{B} \sim 2 n_{c}^{S}$. Ab-initio calculations $[29,30]$ suggested that the electron-phonon coupling constant is on the order of $\lambda_{e-p h} \sim 0.2-0.25$ in this doping range and the rapid increase of $T_{c}$ is consistent with the exponential increase expected by the standard McMillan expression assuming however a reduced $\mu^{*}$ value (on the order of 0.04). As superconductivity vanishes for $k_{F} l \sim 1$ disorder might play a significant role. Indeed, in this regime disorder is expected to hinder the formation of long range phase coherence and localized Cooper pairs could then be preformed in an exotic insulting phase characterized by a small but hard gap (see [28] and discussion therein). However, our study clearly shows that a metallic phase separates the insulating and superconducting states in striking contrast with this model which predicts a direct transition from the superconducting to the insulating states. Finally, the critical temperature was not affected by the epilayer thickness and superconductivity has been observed in both $3 \mathrm{D}$ and $2 \mathrm{D}$ interaction regimes.

The authors would like to thank T. Crozes, S. Dufresnes, B. Fernandez, T. Fournier and G. Julie from the Nanofab platform (Grenoble, France) for their help during the samples contacts preparation. 
[1] Ekimov, V. A. Sidorov, E. D. Bauer, N. N. Mel'nik, N. J. Curro, J. D. Thompson, S. M. Stishov, Nature 428, 6982 (2004).

[2] Y. Takano, M. Nagao, I. Sakaguchi, M. Tachiki, T. Hatano,K. Kobayashi, H. Umezawa and H. Kawarada, Appl. Phys. Lett. 85, 2851 (2004).

[3] E. Bustarret, J. Kacmarcik, C. Marcenat, E. Gheeraert, C. Cytermann, J. Marcus, T. Klein, Phys. Rev. Lett., 93, 237005 (2004).

[4] E. A. Ekimov, V. A. Sidorov, A. V. Rakhmanina, N. N. Mel'nik, R. A. Sadykov, and J. D. Thompson, Sci. Technol. Adv. Mater., 7, S2 (2006); M. Hoesch, T. Fukuda, J. Mizuki, T. Takenouchi, H. Kawarada, J. P. Sutter, S. Tsutsui, A. Q. R. Baron, M. Nagao, and Y. Takano, Phys. Rev. B, 75, 140508, (2007).

[5] N. Dubrovinskaia, L. Dubrovinsky, T. Papageorgiou, A. Bosak, M. Krisch, H. F. Braun, and J. Wosnitza. Applied Phys. Lett., 92, 132506, (2008); E. A. Ekimov, V. A. Sidorov, A. V. Zoteev, J. B. Lebed, J. D. Thompson, and S. M. Stishov. Sci. Technol. Adv. Mater., 9, 044210 (2008); P. Achatz, F. Omnès, L. Ortéga, C. Marcenat, J. Vacik, V. Hnatowicz, U. Köster, F. Jomard and E. Bustarret, Diam. Rel. Mat. 19, 814 (2010).

[6] B. Sacépé, C. Chapelier, C. Marcenat, J. Kacmarcik, T. Klein, M. Bernard, and E. Bustarret. Phys. Rev. Lett. 96, 097006, (2006); K. Ishizaka, R. Eguchi, S. Tsuda, T. Yokoya, A. Chainani, T. Kiss, T. Shimojima, T. Togashi,S. Watanabe, C.-T. Chen, C. Q. Zhang, Y. Takano, M. Nagao, I. Sakaguchi, T. Takenouchi, H. Kawarada, and S. Shin. Phys. Rev. Lett., 98, 047003, (2007); J. Kacmarcik, C. Marcenat, C. Cytermann, A. Ferreira da Silva, L. Ortega, F. Gustafsson, J. Marcus, T. Klein, E. Gheeraert, and Etienne Bustarret. Physica Status Solidi (a), 202, 2160 (2005); M. Ortolani, S. Lupi, L. Baldassarre, U. Schade, P. Calvani, Y. Takano, M. Nagao, T. Takenouchi,and H. Kawarada. Phys. Rev. Lett., 97, 097002 (2006).

[7] A. Kawano, H. Ishiwata, S. Iriyama, R. Okada, T. Yamaguchi, Y. Takano, and H. Kawarada, Phys. Rev. B 82, 085318 (2010)

[8] H. Okazaki, T. Wakita, T. Muro, T. Nakamura, Y. Muraoka, T. Yokoya, S-I Kurihara, H. Kawarada, T. Oguchi and Y. Takano, Appl. Phys. Lett. 106, 052601 (2015).

[9] X.Blase, E.Bustarret, C.Chapelier, T.Klein and C.Marcenat, Nature Mat. 8, 375 (2009)

[10] E. Bustarret, Physica C, 514, 36 (2015).

[11] T. Klein, P. Achatz, J. Kacmarcik, C. Marcenat, F. Gustafsson, J. Marcus, E. Bustarret, J. Pernot, F. Omnès, Bo E. Sernelius, C. Persson, A. Ferreira da Silva, and C. Cytermann Phys. Rev. B 75, 165313 (2007).

[12] Yan-Hsing Lin, J. Nelson, A. M. Goldman Physica C 514, 130 (2015); V.F. Gantmakher and V.T. Dolgopolov, Physics Uspekhi 53, 49 (2010).

[13] G. Zhang,M. Zeleznik, J. Vanacken, P.W. May, and V.V. Moshchalkov, Phys. Rev. Lett. 110, 077001 (2013).

[14] S. Kitagoh, R. Okada, A. Kawano, M. Watanabe, Y. Takano, T. Yamaguchi, T. Chikyow and H. Kawarada,
Physica C, 470, S610-S612 (2010).

[15] A.F. Ioffe and A.R. Regel, Prog. Semicond. 4, 237 (1960).

[16] J. Bousquet, G. Chicot, D. Eon and E. Bustarret, Appl. Phys. Lett. 104021905 (2014).

[17] Note that significantly higher critical temperatures have been reported in epilayers grown on [111]-oriented substrates $[7,8]$. This difference has been attributed to the presence of a large in-plane stress for [111]-grown samples [7] which could significantly alter the topology of the Fermi surface. See J. E. Dijkstra and W. Th. Wenckebach, J. Appl. Phys., 85, 1587 (1999).

[18] A.B. Harker. Journal of Materials Research, 5, 818 (1990).

[19] E.Bustarret, P.Achatz, B. Sacépé, C.Chapelier, L. Oetega and T.Klein, Phil. Trans. R. Soc. A 366, 267 (2008).

[20] E. Gheeraert, S. Koizumi, T. Teraji, H. Kanda and M. Nesladek, physica status solidi (a), 174, 39 (1999).

[21] Taking into account a (small) mass correction factor equal to $\left(m_{h h}^{3 / 2}+m_{l h}^{3 / 2}+m_{s o}^{3 / 2}\right)^{1 / 3} /\left(m_{h h}^{1 / 2}+m_{l h}^{1 / 2}+m_{s o}^{1 / 2}\right) \sim$ $0.5, m_{h h}, m_{l h}$ and $m_{s o}$ being the heavy hole, light hole and spin-orbit effective masses, respectively. See H. Guyot, P.Achatz, A.Nicolaou, P. Le Fevre, F. Bertran, A. Taleb-Ibrahimi, and E. Bustarret, Phys. Rev. B, 92, 045135 (2015).

[22] G. Chicot, A. Fiori,. N. Volpe, T. N. Tran Thi, J. C. Gerbedoen, J. Bousquet, M. P. Alegre, J. C. Piero, D. Araujo, F. Jomard, A. Soltani, J. C. De Jaeger, J. Morse, J. Härtwig, N. Tranchant, C. Mer-Calfati, J. C. Arnault, J. Delahaye, T. Grenet, D. Eon, F. Omnes, J. Pernot, and E. Bustarret, J. of Appl. Phys. 116, 083702 (2014).

[23] Altshuler, B. L., and Aronov, A. G. Electron-Electron Interaction in Disordered Conductors. edited by A.L. Efros and M. Pollak (Amsterdam : Elsevier Scientific Publishing), 1985.

[24] A. Kardakova, A. Shishkin, A. Semenov, G. N. Goltsman, S. Ryabchun, T. M. Klapwijk, J. Bousquet, D. Eon, B. Sacépé, T. Klein, and E. Bustarret, Phys. Rev. B, 93, 064506 (2016).

[25] A. K. Meikap, Y. Y. Chen, and J. J. Lin, Phys. Rev. B, 69, 212202 (2004); Y. L. Zhong and J. J. Lin, Phys. Rev. Lett. 80, 588 (1998).

[26] H.Fukuyama, Physica, 126C, 306 (1984); A.M. Finkel'stein, Physica B, 197, 636 (1994).

[27] M. A. Skvortsov and M.V. Feigel'man, Phys. Rev. Lett., 95, $057002(2005)$

[28] M.V. Feigel'man, L. B. Ioffe, V. E. Kravtsov, and E. A. Yuzbashyan, Phys. Rev. Lett. 98, 027001 (2007).

[29] Y. Ma, J. S. Tse, T. Cui, D. D. Klug, L. Zhang, Y. Xie, Y. Niu, and G. Zou. Phys. Rev. B, 72, 014306 (2005); L. Boeri, J. Kortus, and O. K. Andersen. Phys. Rev. Lett., 93, 237002 (2004); K. Kadas, L. Vitos, and R. Ahuja. Applied Physics Letters, 92 052505, (2008).

[30] H. J. Xiang, Z. Li, J. Yang, J. G. Hou, Q. Zhu. Phys. Rev. B, 70, 212504 (2004); X. Blase, Ch. Adessi, and D. Connétable Phys. Rev. Lett., 93237004 (2004). 